testosterone slows the rate of growth of the left hemisphere and suppresses the development of the thymus gland. They thereby attempt a unified explanation of the apparent over-representation of males and left-handers who present with developmental learning disorders and certain auto-immune diseases. This left hemisphere retardation may alter the brain's pattern of connectivity as competition for synaptic space takes place in the developing cortical plate, a process for which Goldman-Rakic and Rakic have provided an elegant experimental analysis in monkey. Nonetheless, one would like to see Geschwind and Behan's epidemiological observations replicated by independent investigators. In the past, left-handers have proved a notoriously unreliable group with respect to almost any measure of neuropsychological functioning one can think of. Curiously, Geschwind and Behan fail to mention the traditional story that lefthanders are, on average, poor at visuospatial skills, for which right-hemisphere pathology would be implicated; they also ignore Kimura's recent evidence (Human Neurobiology 2, 147-154; 1983) that lefthanders, as a group, are just as left-dominant for speech representation as righthanders.

The best animal analogue of human vocal learning is to be found in bird-song. Song learning in canaries is, like human speech, "a left-sided affair" (Nottebohm). Lesion of the left hypoglossus nerve, which controls the left half of the syrinx, has a far more deleterious effect upon singing than does lesion of the right. More surprisingly, seasonal variation in the pattern of song appears to be determined by the growth of new neurones and synapses. Although there is currently no evidence for such synapto- and neuro-genesis in the adult human brain, Nottebohm speculates that:

In the not-so-distant future it may be possible to treat local regions of the brain to activate genes that induce dendritic retraction and growth, that induce synaptic formation and shedding, that induce birth, migration, and differentiation of new neurons.

At present, Nottebohm's “'hope for a new neurology" is science-fiction although the advances of the past decade do raise the serious possibility that the diagnosis of "irreversible" brain damage will eventually give way to a less horrendous prognosis. Such success would be a fitting tribute to Professor Geschwind, who died at a tragically early age last year. The emerging synthesis that this book displays can in large part be credited to the enthusiasm, dedication and insight with which Norman Geschwind always insisted that cerebral dominance does have demonstrable biological foundations.

John C. Marshall is in the Neuropsychology Unit part of the Neuroscience Group at the Radcliffe Infirmary, Oxford.

\section{All keyed up in the laboratory}

\section{E.G. Richards}

Computers in Biology: An Introduction. By Robert Ransom and Raymond $\mathbf{J}$. Matela.

Open University Press, Milton Keynes/ Taylor \& Francis, Philadelphia: 1985.

Pp.150. £9.95, \$19.

Microcomputers in Biology: A Practical Approach.

Edited by C.R. Ireland and S.P. Long. IRL: 1984. Pp.324. Pbk £15, \$27.

Mathematics, Biology and

Microcomputers.

By A.N. Barrett.

Elsevier-Biosoft: 1984. Pp.102. £24, \$36.

THE day has not quite arrived when a biologist, unaccustomed to computers, can use one as he might use a centrifuge. He must go through the laborious and daunting process of coming to terms with whatever computer is available to him, its jargon, its hardware and its capabilities, its operating system and more besides. Having done that he then must either set about learning its language and write a program for the task he has in mind (and in the process more than likely reinvent the wheel) or, if he is lucky, use software written by somebody else.

Even then he is likely to encounter many pitfalls: the program may require modification before it will run on his computer (even if it is written in a language familiar to his machine); the documentation may be inadequate or even absent; it may lack robustness and die on him with an obscure error message as a result of a mis-key after he has been entering data for the last hour; he may be lulled into a false sense of security by a program which presents him with the unadorned slope of a straight line fitted to data points which are scattered at random.

Help in coming to terms with all this is provided by Ransom and Matela in Computers in Biology, a short but comprehensible account of some aspects of computer science. The book first explains how computers might be useful and what is involved in using them. It goes on to introduce data structures, lists and trees and the like, and then to discuss statistical analyses using computers, computer graphics, interfacing experimental apparatus with computers and finally the simulation of models for biological processes. One appendix is used to describe briefly computer hardware as we know it today, and discuss how to choose a computer (including a section on how to deal with computer salespeople), while another introduces operating systems and the facilities they might offer. A useful glossary is also provided.

In a book of such generality, no program listings are included but the text is illustrated with many examples of how computers might be applied in various branches of biology. Even if it does not cover your pet subject, it may give you seminal ideas.

Not every research worker has access to a mainframe or a mini, and may have to contemplate choosing a microcomputer. Assistance in this confusing task is at hand in Microcomputers in Biology, a multiauthored work edited by Ireland and Long. The first chapter goes some way to explain some of the jargon, also offering advice on choosing a microcomputer, and the book continues with chapters on the mysteries of interfaces, the problems involved in datalogging and the possibilities inherent in computer graphics. These are useful epitomes for workers with some experience in computing but may present problems to the complete beginner. The book includes summaries of some ways in which microcomputers can be used to control apparatus or analyse data obtained from several analytical techniques: spectrophotometry, chromatography and ultracentrifugation, among them. It ends with a chapter on controlling environmental factors. There is also a chapter on doing various things with nucleic acid sequences, which, it should be noted, does not attempt to come to terms with using sequence databases (such as is available from EMBL) with microcomputers.

The book should appeal to biologists who use such techniques and have some flair for instrumentation, and who are contemplating using a microcomputer. Most chapters end with listings of programs, usually in BASIC, which run on the author's microcomputer (discs containing versions of these programs are also to be available for four popular microcomputers). It is unclear how easy these will be to use, how robust they are or to what extent they protect the user from drawing false conclusions.

Barrett's Mathematics, Biology and Microcomputers also provides listings of simple programs (for instance to solve quadratic equations, though many of them would be better tackled on a hand calculator) but most of his book is taken up with an introduction to BASIC, solving quadratic and cubic equations (but stopping short of iterative methods for solving general equations), matrices, coordinate geometry, curve fitting, simple mensuration and so on. It is essentially an introduction to certain parts of mathematics and as such reflects, as do the biological examples, what are presumably the author's interests. It ends with a somewhat mysterious section on the manipulation of biological data by prime numbers. Unfortunately the reference to further details of this is still in press.

E.G. Richards is Visiting Lecturer at the Department of Biophysics, King's College, University of London. 